Radioprotection, DunOD, 1980

Vol. $15, \mathrm{n}^{\circ} 1$, pages $23-40$

\title{
Les bases biologiques de la radioprotection $\left(^{*}\right)$
}

\author{
M. TUBIANA $(* *)$ \\ (Manuscrit reçu le 16 novembre 1979)
}

\begin{abstract}
RÉSUMÉ
La radioprotection est basée sur un grand nombre de données humaines qui ont été recueillies au cours des 80 dernières années. Pour des niveaux de doses de quelques centaines de rads, les risques peuvent être évalués avec une remarquable précision. Toutefois, il est difficile d'en déduire les risques provoqués par de faibles doses, en raison de l'incertitude sur la forme de la relation dose-effet. La pratique adoptée consiste à se baser sur des hypothèses pessimistes, donc à surestimer ces risques. Néanmoins, même dans ces conditions défavorables, les risques liés aux activités professionnelles impliquant une exposition à des rayonnements paraissent moins importants que dans la plupart des industries.

La radioprotection a eu un rôle historique essentiel dans l'estimation quantitative des risques et a inauguré une nouvelle ère de la médecine du travail et de l'étude des maladies dues à l'environnement. De nombreuses substances, comme les rayonnements, sont mutagènes et/ou cancérogènes à de très faibles doses et, dans de très nombreux cas, l'homme ne peut éviter d'y être exposé. Par conséquent, la politique de refus de tout risque et de la sécurité absolue fait miroiter des espérances irréalisables et fallacieuses. La seule méthode est de déterminer l'importance quantitative des divers risques afin de pouvoir décider du degré de dommage qui est tolérable dans les différents cas où une exposition est inévitable.
\end{abstract}

\begin{abstract}
Radiation protection is based on a large number of human data collected during the past 80 years. For dose levels of a few hundred rads, risks can be evaluated very accurately. Yet it is difficult to derive from them the risks due to low doses because of the uncertainty on the dose-effect relationship. In the practice, pessimistic assumptions are used, which involves an over-estimation of risks. However, even in these unfavorable conditions, risks associated to occupational activities implying radiation exposure seem to be less important than in most industries. Radiation protection has played a historical and essential part in the quantitative assessment of risks and opened a new era of occupational medicine and environmental health investigations. Many
\end{abstract}

(*) Communication présentée à la Conférence de l'Uranium Institute en septembre 1979.

(**) Institut Gustave-Roussy, Villejuif, 16 bis, avenue Paul-Vaillant-Couturier, 94800 Villejuif.

RADIOPROTECTION, VOL. 15 - 0033-8451/1980/23/\$ 5.00/C Bordas-Dunod. 
substances, such as radiations, are mutagenic and/or carcinogenic at very low doses, and in many cases human exposure cannot be avoided. Therefore, a policy advocating refusal of any risk whatsoever and absolute safety will lure with unattainable and misleading prospects. The only method is to assess the quantitative importance of the various risks in order to decide how far a damage may be tolerable in the various cases when exposure cannot be avoided.

Les rayons X furent découverts par Roentgen en 1895 et la radioactivité naturelle par Becquerel en 1896. Les effets délétères, pour l'homme, des rayonnements émis par des substances radioactives ont été décrits dès 1897.

L'histoire de cette découverte mérite d'être contée. Henri Becquerel remarqua un jour un érythème sur sa poitrine. Cherchant quelle pouvait en être la cause, il se souvint qu'il avait transporté quelques jours auparavant dans la poche de son gilet des substances radioactives destinées à Pierre Curie pour des travaux qu'ils effectuaient en commun. Il parla de sa découverte à Pierre Curie et décida, pour vérifier son hypothèse, de garder pendant le même temps un peu de cette substance, mais cette fois-ci dans l'autre poche de son gilet. Quelques jours après, il observa un érythème sur sa poitrine de l'autre côté. Pierre Curie décida alors de se livrer à la même expérience et plaça pour ce faire la substance successivement dans ses deux poches. Ils acquirent ainsi la certitude que l'érythème était d'origine radiobiologique et relatèrent leur découverte.

Le premier cancer radio-induit chez l'homme a été observé en 1902. Pierre Marie en 1910 fut le premier à apporter la preuve chez l'animal de l'effet cancérogène des rayonnements. Depuis lors, de très nombreuses données ont été recueillies et le rapport de 1977 du Comité des Nations Unies sur les effets des rayonnements ionisants (U.N.S.C.E.A.R.), qui se présente sous la forme d'un document de plus de 700 pages, n'est qu'un bref résumé incomplet des publications les plus récentes bien qu'il compte plusieurs milliers de références [16]. Plusieurs autres organismes internationaux et nationaux comme le British Medical Research Council ou l'Académie Nationale des Sciences des États-Unis (B.E.I.R.) ont également créé des commissions chargées d'effectuer des analyses approfondies des connaissances acquises à ce jour sur ce problème. Tous ces rapports aboutissent heureusement à des conclusions voisines.

D'autre part, la Commission Internationale de Protection Radiologique (C.I.P.R.) a publié régulièrement, depuis sa création en 1928, des mises à jour complètes ainsi que des recommandations basées sur les informations disponibles [6]. Ces dernières ont toujours été acceptées par tous les pays et le rôle de la C.I.P.R. est universellement reconnu. Elle a publié en 1977 son $26^{\mathrm{e}}$ rapport auquel je me référerai souvent dans cet exposé [7].

Nos connaissances sur les effets tardifs des rayonnements ionisants sont le fruit de l'étude de populations exposées à des doses connues de rayonnements et sur lesquelles les conséquences d'une irradiation ont été étudiées scientifiquement. Il s'agit, notamment, de plusieurs dizaines de milliers 
de malades traités par des rayons $\mathrm{X}$ ou des radio-isotopes pour des affections bénignes, de plusieurs groupes de travailleurs (ouvriers qui peignaient des cadrans lumineux avec une peinture au radium, radiologistes, mineurs dans des mines d'uranium, ...) et des 285000 survivants d'Hiroshima et Nagasaki qui ont été suivis depuis 1945 par une équipe de 500 spécialistes créée conjointement par le Japon et les États-Unis [5, 16].

Les effets biologiques tardifs des rayonnements ionisants peuvent être classés en trois catégories [7, 16].

1. Les effets différés sur les tissus irradiés : par exemple baisse de la fécondité et stérilité, cataractes ou diminution de la croissance des enfants. Lorsque les doses sont assez élevées (au-dessus d'un seuil de quelques centaines ou milliers de rads), ces effets sont observés chez tous les sujets irradiés et leur gravité augmente avec la dose.

2. Les effets cancérogènes : on a observé chez les sujets exposés à des hautes doses d'irradiation une fréquence accrue des cancers. Ces cancers ressemblent à ceux qui apparaissent spontanément et on ne peut les en distinguer. Après des doses de rayonnements même élevées, la probabilité d'un cancer radioinduit reste faible, de l'ordre de quelques pour cent. L'ampleur de cet effet aléatoire croît avec la dose. Comme nous le verrons plus loin, on connaît encore mal la forme mathématique de la relation dose-effet. En outre l'existence d'un seuil est encore controversée.

3. Les effets génétiques : on sait depuis plus de 50 ans que les rayonnements provoquent des mutations. Les généticiens ont constaté que presque toutes les mutations sont délétères ou, dans le meilleur des cas, neutres. Les gènes les plus courants dans une population ont été passés au crible de la sélection naturelle et les gènes mutants ont peu de chance d'être aussi bien adaptés qu'eux.

La mutation, comme la cancérogénèse, est un effet aléatoire, dit stochastique, et l'on admet de façon générale qu'il n'existe pas de seuil.

Les premières recommandations de la C.I.P.R. étaient basées principalement sur les effets somatiques non stochastiques des rayonnements et le concept de dose admissible reposait sur l'existence d'un seuil [6]. Une dose était jugée acceptable si elle était suffisamment basse pour ne pas avoir d'effet nocif décelable. Plus tard, au début des années 50 , à un moment où j'étais membre de la C.I.P.R., on accorda une plus grande attention au pouvoir cancérogène des faibles doses. Il apparut alors clairement que les effets aléatoires sans seuil - à savoir la cancérogénèse ou la mutagenèse - étaient les plus graves dangers des faibles doses d'irradiation. Les spécialistes de la radioprotection durent donc mettre l'accent sur l'évaluation de ces risques.

Ce n'est pas là une situation unique; pour l'amiante, par exemple, la protection a suivi la même évolution. Au début, l'asbestose était considérée comme le principal danger; le but de la médecine du travail était de ramener l'exposition à la poussière d'amiante à un niveau inférieur à celui qui provoquait cette maladie. Plus tard, on découvrit que lorsque les travailleurs ne souffraient plus d'asbestose, on constatait chez eux un risque d'apparition

vOL. $15-\mathrm{N}^{\circ} 1$ 
de cancers du poumon ou de mésothéliomes légèrement plus élevé que dans la population en général. Il fallait donc établir les mesures de protection en tenant compte de la relation entre la concentration d'amiante dans l'air et la probabilité d'apparition d'un cancer.

\section{ÉVALUATION DU RISQUE DE CANCER}

Pour chiffrer les effets cancérogènes des rayonnements, on s'est servi de plusieurs enquêtes épidémiologiques et tout particulièrement des études prolongées et minutieuses sur les survivants de la bombe A, qui sont maintenant suivis depuis plus de 30 ans [5, 16]. Sur les 285000 survivants enregistrés à Hiroshima et Nagasaki, 80000 sont décédés de mort naturelle entre 1950-1978. On estime qu'approximativement 400 à 500 de ces morts étaient dues à un cancer radio-induit. Sur les 1200 survivants ayant reçu les doses les plus fortes (dose moyenne de 330 rads, soit très vosines de la dose létale 50 que l'on situe entre 350 et 450 rads environ), l'augmentation de la fréquence des leucémies entre 1950 et 1974 était de $1 \%$. Ainsi, l'effet cancérogène est indubitable mais, en termes absolus, le nombre des survivants atteints est relativement petit et ne modifie pas de façon significative le taux de survie global de la population ayant été exposée à la bombe A. Notons, à ce propos, que ces études n'ont mis en évidence aucune maladie particulière due à l'irradiation hormis les opacités critalliniennes et un léger retard du développement staturo-pondéral chez les enfants exposés très jeunes. On n'a, en particulier, observé ni d'augmentation de la morbidité, ni une quelconque accélération du processus de vieillissement, ni un raccourcissement de la durée de vie en dehors de celui qui est directement lié à la fréquence accrue des cancers [5].

La dose reçue par chacun des survivants a été déterminée avec précision en tenant compte notamment de la distance à laquelle celui-ci se trouvait par rapport à l'hypocentre au moment de l'explosion. Toutefois, la nature des rayonnements reçus par les habitants des deux villes est différente. La bombe d'Hiroshima était composée d'uranium 235, un tiers environ de l'irradiation était dû à des neutrons et le reste à des rayons gamma.

Comme l'efficacité biologique relative (E.B.R.) des neutrons est d'environ 10, $80 \%$ de l'effet biologique a été dû aux neutrons. En revanche, à Nagasaki la bombe en plutonium 239 était de conception différente et libérait presque uniquement des rayons gamma. Cette différence justifie une analyse séparée des données relatives à Hiroshima et Nagasaki et leur comparaison informe directement sur les effets cancérogènes relatifs des neutrons et des photons sur l'espèce humaine.

Dans ces deux villes, l'effet cancérogène des rayonnements se manifesta d'abord par une augmentation du nombre des leucémies à la fin des années 40 . Après être passé par un pic de 1952 à 1970, le taux de leucémie ne cessa de diminuer tandis qu'augmentait la fréquence des tumeurs solides radioinduites, essentiellement de la thyroïde, du sein et des poumons. La période de latence entre le moment de l'exposition et l'apparition du cancer varie 
selon la nature de la tumeur et l'âge du sujet au moment de l'exposition : pour les leucémies aiguës elle est de 5 à 10 ans chez des sujets irradiés dans l'enfance, et de 10 à 15 ans chez ceux irradiés à l'âge adulte. Pour les tumeurs solides, le temps de latence varie entre 15 et 30 ans; il peut être encore plus long dans le cas des myélomes multiples et des cancers des voies digestives. On a constaté, d'autre part, que l'effet cancérogène varie en fonction de l'âge. Pour de nombreux cancers, le risque relatif est plus élevé chez les sujets exposés avant l'âge de 20 ans. Le taux de leucémie aiguë est plus élevé chez des sujets irradiés à moins de 10 ans ou à plus de 50 ans. En ce qui concerne le cancer du sein, le risque relatif est maximal chez des femmes âgées de 10 à 19 ans au moment de l'irradiation.

Jusqu'en 1975, les leucémies représentaient environ un tiers des tumeurs malignes. Elles offraient donc le meilleur échantillon pour une étude quantitative des effets cancérogènes. A Nagasaki, la fréquence des leucémies dans le groupe ayant reçu une dose supérieure à 100 rads est nettement plus élevée que chez les sujets témoins. Au-delà de cette dose, la fréquence des leucémies augmente avec la dose. L'incidence accrue des leucémies observée chez les survivants de la bombe correspond à un taux d'induction d'environ 20 à 30 leucémies pour $10^{4}$ sujets ayant reçu 100 rads. Chez les survivants d'Hiroshima, le taux d'induction semble plus élevé; en effet, on a enregistré 50 cas par $10^{4}$ sujets exposés à 100 rads; en outre, on n'a aucune preuve de l'existence d'un seuil.

D'autres études peuvent servir à effectuer une évaluation quantitative des radio-leucémies [7, 16, 17]. Parmi celles-ci, la plus importante est l'étude réalisée par Court-Brown et Doll sur 20000 patients qui avaient subi une ou plusieurs séries de radiothérapies pour des spondylarthrites ankylosantes. Court-Brown et Doll n'ont décelé aucun effet pour des doses inférieures à 300 rads. En revanche, pour des doses plus élevées, l'incidence augmente avec la dose. Chez la plupart des malades, $50 \%$ de la moelle osseuse avait été irradiée; si l'on admet que cette irradiation équivaut à une dose moyenne moitié plus faible en irradiation totale, le taux d'induction correspond à celui calculé pour les cas de Nagasaki. Des recherches effectuées sur des malades traités par irradiation de la zone pelvienne ou du cuir chevelu ou sur des radiologues irradiés dans le cadre de leur activité professionnelle entre 1920 et 1940 aboutissent à des résultats similaires. Dans ce dernier cas, les observations suggèrent que des irradiations fractionnées, même si elles s'étendent sur de longues périodes, ont un pouvoir leucémogène voisin de celui d'une irradiation aiguë. De même, le fait que les estimations du risque de leucémies soient presque identiques, que la moelle osseuse ait été totalement ou seulement en partie irradiée, semble indiquer qu'une dose donnée produit pratiquement le même effet lorsqu'elle est répartie uniformément que si elle ne l'est pas. Toutefois, il convient de souligner que toutes ces données sont basées sur des observations effectuées sur des sujets ayant reçu des doses supérieures à 100 rads.

Des estimations approximatives semblables ont été obtenues pour une douzaine de tissus ou organes différents; pour bon nombre d'entre eux, 
et en particulier la thyroïde, le sein, les poumons, les os, différentes enquêtes fournissent des estimations qui paraissent concordantes dans les limites de précision des études effectuées [7, 16]. Pour déterminer le risque global d'apparition d'une tumeur maligne après irradiation de l'organisme entier, on peut faire l'hypothèse que les risques pour les divers tissus ou organes s'ajoutent. De plus, plusieurs études ont comparé la fréquence de l'ensemble des tumeurs malignes par rapport à celle des seules leucémies; ce rapport varie de 3 à 5 . Sachant que le risque de leucémie se situe aux alentours de $2.10^{-3}$ pour $100 \mathrm{rads}$, le risque total de cancer devrait être de l'ordre de $10^{-2}$ pour 100 rads. Cette estimation concorde assez bien avec celle obtenue en additionnant les risques pour chaque tissu. Précisons, une fois encore, que toutes ces valeurs correspondent à des effets cancérogènes induits par des doses supérieures à 100 rads.

Pour la radioprotection, ce sont les faibles doses - d'approximativement quelques rads - qui présentent le plus grand intérêt. Il faut donc, en radioprotection, évaluer l'effet cancérogène de quelques rads sur la base des chiffres obtenus pour des doses d'une ou de plusieurs centaines de rads. Certains résultats suggèrent que des doses modérées de rayons $\mathrm{X}$ - quelques dizaines de rads - provoquent des cancers; néanmoins, compte tenu de l'importante incertitude statistique liée aux données pour de tels niveaux d'irradiation, on ne peut pas se fier à ces résultats pour évaluer la fréquence d'induction après de telles doses.

Une augmentation de la fréquence des leucémies et/ou d'autres tumeurs malignes a été signalée dans certaines études chez des adultes ayant reçu des doses assez faibles au cours de leurs occupations professionnelles ou pendant des examens radiologiques. Parmi les études consacrées à ce problème, la plus connue est celle de Mancuso. Néanmoins, la méthodologie adoptée pour ces recherches et leurs résultats ont été sévèrement critiqués. Il semble aujourd'hui peu probable que ce type d'investigation puisse apporter des éclaircissements sur les effets des faibles doses d'irradiation. En outre, les calculs présentés dans le rapport du Comité des Nations Unies montrent que, à moins que les estimations actuelles du taux d'induction total de cancer soient grossièrement erronées, il faudrait effectuer des enquêtes portant sur des travailleurs soumis à des doses annuelles moyennes sur l'organisme entier de plus d'un rad pendant des millions d'homme-ans pour pouvoir évaluer directement le risque de cancer consécutif à ces doses. Il y a donc peu d'espoir de parvenir un jour à recueillir des données fiables sur les effets des faibles doses. Nous devons donc déduire les estimations à partir des informations obtenues pour des doses beaucoup plus élevées. Ce type d'extrapolation exige que l'on connaisse la forme de la relation dose-effet ainsi que les valeurs absolues des coefficients qui y figurent. En réalité, on a peu de renseignements sur la forme de la relation dose-effet. Chez les survivants de Nagasaki, la courbe dose-effet pour des doses supérieures à 100 rads n'est pas linéaire ( fig. 1) car l'effet leucémogène par unité de dose est deux fois plus élevé pour une dose moyenne de 330 rads qu'il ne l'est pour une dose moyenne de 100 rads [16]. Cette diminution de l'effet par rad à mesure que diminue la 
dose n'a pas été observée à Hiroshima. Dans cette ville, qui a été irradiée surtout par des neutrons, la courbe dose-effet est linéaire sur toute la gamme des données existantes ( fig. 2). Toutefois, il convient de souligner que, dans les deux villes, le nombre d'observations recueillies n'a pas une précision statistique suffisante pour permettre de se prononcer définitivement sur la forme mathématique de ces courbes.

D'autres données, notamment celles concernant les cancers de la thyroïde et du sein, peuvent aussi bien être représentées par une relation quadratique que linéaire. Ces informations ne permettent donc pas d'exclure l'une ou l'autre de ces courbes dose-effet.

Quelles informations peut-on espérer obtenir des recherches fondamentales et des observations expérimentales, qui puissent guider dans ce choix de la relation dose-effet?

Il est généralement admis qu'une tumeur est le résultat d'une succession d'événements distincts. L'étude des cancers provoqués par des produits chimiques révèle qu'on doit distinguer au moins deux étapes indépendantes : l'initiation et la promotion. L'initiation est un processus irréversible rapide par lequel une cellule normale acquiert des caractéristiques néoplasiques. Mais la cellule « initiée », ou " transformée ", peut rester indéfiniment à l'état " quiescent " sans proliférer. La promotion est le mécanisme par lequel une cellule transformée donne naissance à une tumeur capable de croître.

Bien que les rayonnements ionisants aient à la fois des propriétés d'initiateur et de promoteur, il est utile de distinguer ces deux étapes. L'initiation intervient probablement dans le matériel génétique de la cellule [9]. Il est donc justifié de se référer aux courbes dose-effet obtenues pour des systèmes cellulaires simples.

La mutation d'une fleur, la tradescantia (ou éphémère de Virginie), est un processus simple sur lequel nous disposons de nombreuses données quantitatives. Les effets obtenus pour des doses de rayons $X$ allant jusqu'à 100 rads sont bien représentées par une fonction du type $I=\alpha D+\beta D^{2}$ [15]. A faible dose, la composante linéaire de la courbe prédomine; il y a proportionnalité entre la dose et les effets mais la probabilité de l'événement est extrêmement faible. Pour des doses supérieures à quelques dizaines de rads, la composante quadratique $\left(\beta \mathrm{D}^{2}\right)$ prédomine (fig. 3). Des relations dose-effet semblables ont été obtenues dans des travaux consacrés aux aberrations chromosomiques radio-induites dans des cellules humaines cultivées in vitro [10] (fig. 4).

Dans ces deux cas, la composante linéaire apparaît seule, avec le même coefficient $\alpha$, lors d'irradiations avec de faibles débits de dose. La composante quadratique semble être due à l'effet cumulé de deux lésions réversibles. Lors d'une irradiation à faible débit de dose, une lésion réversible a toutes les chances d'être réparée complètement avant l'événement suivant et, de ce fait, ne contribue pas à créer une lésion irréversible.

Lors d'irradiations avec des particules lourdes, comme les neutrons ou les rayons $\alpha$, on n'observe qu'une composante linéaire mais sa pente

voL. $15-\mathrm{N}^{\circ} 1$ 
est beaucoup plus forte. Ceci signifie que la probabilité d'une lésion irréversible par unité de dose absorbée est beaucoup plus importante.

La théorie du double effet de Rossi-Kellerer explique bien ces phénomènes. D'après cette théorie, l'effet biologique primaire n'intervient que si deux événements se produisent dans le volume sensible dans un intervalle de temps relativement court. Dans le cas des particules lourdes, l'énergie est cédée dans les tissus sur de courtes trajectoires le long desquelles l'ionisation est dense. Par conséquent, lorsqu'une particule caractérisée par une ionisation dense traverse le volume sensible d'une cellule, elle a de fortes chances d'entraîner les deux événements.

Dans le cas des rayons $\mathrm{X}$, en revanche, le transfert d'énergie le long de la trajectoire parcourue par la particule est bien moindre, et il faut donc, en général, deux particules pour que se produisent les deux événements qui provoquent la lésion biologique (composante quadratique). Toutefois, du fait des fluctuations de l'énergie déposée le long de la trajectoire, il existe une faible probabilité pour qu'une particule libère suffisamment d'énergie dans le volume sensible. Cette possibilité explique la composante linéaire de la courbe.

Cette théorie s'accorde remarquablement bien avec l'influence de la dose et du débit de dose pour les divers types de rayonnements [1]. Le fait qu'il existe une bonne corrélation entre cette théorie et les résultats expérimentaux semble indiquer que celle-ci est valable, sans toutefois le prouver de façon irréfutable. D'après elle, la relation dose-effet devrait être curvilinéaire pour les cancers induits par les rayons $\mathrm{X}$, et linéaire pour ceux dus aux neutrons [1]. Ceci concorde avec la différence observée, pour les leucémies, entre Hiroshima et Nagasaki. Mais ce fait n'est pas non plus, à lui seul, une preuve parfaitement convaincante.

En réalité, la cancérogenèse constitue l'aboutissement d'un processus complexe et rien ne permet d'affirmer avec certitude que la relation dose-effet devrait être pour elle décrite par le même type de fonction que l'effet observé au niveau de la cellule. Pour diverses raisons, telles que l'absence de promotion, les relations entre l'hôte et la tumeur qui mettent en jeu les défenses immunitaires, la possibilité d'une période de latence supérieure à l'espérance de vie ou le fait que de nombreuses cellules doivent être modifiées pour qu'un cancer se développe, il est possible qu'après de faibles doses, l'effet par rad soit inférieur à ce qu'il devrait être d'après la relation dose-effet pour les lésions cellulaires. En outre, aucun système cellulaire étudié in vitro n'est à l'heure actuelle parfaitement adapté à l'étude de l'induction des tumeurs.

Ces considérations montrent à quel point il est difficile d'effectuer des prévisions sur la base des systèmes cellulaires in vitro et soulignent l'intérêt de l'expérimentation animale.

L'apparition des tumeurs après irradiation a été beaucoup étudiée chez les animaux. Pour les tumeurs consécutives à une exposition à des rayons $\mathrm{X}$, l'effet par unité de dose absorbée décroît avec le débit de dose [16]; il n'en n'est pas de même lorsque l'irradiation est due à des particules lourdes. 
Pour la plupart des tumeurs observées chez les animaux (leucémies, tumeurs des reins, de la peau et du poumon), les données sur la cancérogenèse provoquée par de faibles doses de rayons X peuvent être représentées par une relation polynomiale du second degré et celles sur les cancers induits par les particules lourdes par une relation linéaire sans seuil. Cependant, les courbes dose-effet ont des formes mathématiques variables selon les tumeurs et les animaux d'expérience. Ainsi, certaines tumeurs semblent pouvoir être décrites par une relation quadratique entre la dose et l'effet, ou par une courbe dose-effet linéaire avec seuil ou quasi-seuil. Il existe plusieurs exemples d'une diminution de l'incidence des tumeurs aux faibles doses alors que l'on a obtenu dans un seul cas (néoplasie mammaire chez le rat Sprague-Dawley) une courbe linéaire sans seuil après exposition aux rayons $X$. Précisons, néanmoins, que même dans ce cas la relation s'écarte de la fonction linéaire aux plus faibles doses [16]. Cependant, ces contradictions ne sont pas surprenantes étant donné la diversité et la complexité des processus cancérogènes.

En résumé, bien que les informations soient très nombreuses et fournissent une évaluation satisfaisante du risque provoqué chez l'homme par de fortes doses ou des débits de dose élevés, elles ne permettent pas de parvenir à une conclusion définitive en ce qui concerne la forme de la relation doseeffet ni l'effet après de faibles doses de rayons X. Des analyses théoriques ainsi que les données sur des systèmes simples suggèrent que la relation du type polynominal du second degré est la plus probable, mais elles ne fournissent pas de valeur numérique pour les coefficients. Les informations dont nous disposons sur la radiocancérogenèse chez les mammifères confirment la validité du modèle polynominal du second degré pour l'exposition aux rayons X. Mais il est difficile de dire dans quelle mesure l'effet, par unité de dose absorbée, diminue pour des faibles doses ou débits de doses. Cette diminution varie en fonction du système biologique entre 1 et 10 environ $[2,3]$. En outre, les résultats d'expériences qui montrent que la courbe dose-effet n'a pas le même aspect pour toutes les tumeurs observées chez les animaux prouvent à quel point il est difficile de généraliser.

Aussi, bien que les données tendent à montrer que la relation polynomiale du second degré est celle qui décrit le mieux le risque de cancer, il est vraisemblable que, pendant quelques temps encore, on continuera à estimer celui-ci au moyen d'une relation linéaire sans seuil. Cette politique est logique puisqu'il s'agit d'évaluer un risque, situation dans laquelle il est préférable de surestimer un effet nocif plutôt que de le sous-estimer. Or, comme l'ont montré les spécialistes de l'U.N.S.C.E.A.R. et de la C.I.P.R., les relations linéaires sans seuil ont tendance, pour les faibles doses, à surestimer l'effet $[7,16]$.

Notons, à ce propos, que ces faits suggèrent que la valité du concept de dose collective est contestable. La dose collective est égale au produit de la dose individuelle moyenne par le nombre de personnes exposées; elle est donc identique, que 100 individus aient été exposés à 10 rads ou 10000 à $0,1 \mathrm{rad}$. Étant donné les relations les plus probables entre la dose et l'effet, il faudra sans doute reconsidérer les limites de l'utilisation de ce concept.

VOL. $15-\mathrm{N}^{\circ} 1$ 


\section{ÉVALUATION DU RISQUE GÉNÉTIQUE}

Il est encore plus difficile d'évaluer le risque génétique que le risque de cancer. Je passerai rapidement sur celui-ci car, au cours des dernières années, les risques somatiques ont joué un rôle plus important dans l'élaboration des normes de radioprotection que les risques génétiques.

Comme nous l'avons vu, le matériel génétique des cellules peut être irréversiblement lésé sous l'effet d'une irradiation. Si ces mutations interviennent dans les cellules germinales, elles sont transmises aux descendants des personnes irradiées.

Nous disposons de peu d'informations sur les effets de l'irradiation sur l'homme, qui puissent permettre d'estimer le risque génétique [16]. Il est important de noter que les études d'Hiroshima et de Nagasaki ont été négatives sur ce point [5]. Les données recueillies sur les malformations congénitales, la morphologie et la survie n'ont fait apparaître aucune différence entre les enfants de parents irradiés et ceux du groupe témoin. Dans d'autres études, plus de 100 générations de souris ont été exposées à raison de 200 rads par génération sans que l'on ait pu détecter de répercussion sur la viabilité de leurs portées ou leur fécondité. Les résultats de ces irradiations intensives sont donc rassurants [4].

Pour évaluer le risque génétique d'une irradiation, de nombreuses recherches ont mesuré la fréquence, par unité de dose, de divers types de mutations génétiques provoquées sur la souris et sur des insectes, en particulier la mouche à vinaigre, ou drosophile. La comparaison avec les études récentes effectuées sur des cultures de cellules humaines suggère que la relation dose-effet au niveau cellulaire a la même forme chez toutes les espèces [10].

Les mutations sont usuellement classées en mutations dominantes ou récessives. Une mutation dominante s'exprime totalement chez le descendant même si elle n'est transmise que par un parent. Un gène récessif hérité d'un parent ne se manifeste pas chez le descendant à moins que ce dernier n'ait reçu de l'autre parent le même gène portant la même mutation. Cette distinction est cependant trop schématique. Au cours des dernières décennies, des informations se sont accumulées qui prouvent que des gènes récessifs peuvent avoir des effets délétères et influer sur l'organisme même s'ils ne s'expriment pas complètement parce qu'ils sont hétérozygotes [4].

L'U.N.S.C.E.A.R., la C.I.P.R. et le B.E.I.R. [17] ont récemment analysé chacun de son côté l'énorme masse d'informations sur les effets génétiques existant à ce jour. Leur conclusions, quoique n'étant pas identiques, se recoupent.

Les études de l'U.N.S.C.E.A.R. et du B.E.I.R. $[16,17]$ estiment le nombre de lésions induites à un peu moins de 200 dans la descendance d'un million de sujets ayant reçu 1 rad (après irradiation par rayons X) dont peut-être le quart ou la moitié s'exprimeraient à la première génération et le reste dans les 10 à 15 générations suivantes. Les conclusions de l'analyse critique de la C.I.P.R. aboutissent à une approximation voisine pour l'ensemble 
des générations. Lorsque l'on considère les dangers auxquels est exposé un individu on peut penser que le dommage génétique qui l'affectera le plus est le risque d'apparition d'anomalies chez ses enfants, petits-enfants et arrière-petits-enfants. Ce risque serait en moyenne de 30 à 40 tares génétiques par million d'enfants après une exposition des enfants à $1 \mathrm{rem}$, soit environ un tiers du chiffre obtenu pour le risque somatique de cancer. Pour estimer le risque, il faut, là encore, le comparer à la fréquence dite naturelle qui est de 107000 par million.

En estimant le danger par homme-rad réparti sur une population active de 20 à 65 ans, ou sur la population en général, on doit se rappeler que les deux tiers des hommes-rads n'auront pas d'effet génétique nuisible car ils atteindront des individus qui n'auront, de toute façon, plus d'enfant. Il convient, de même, de noter que nous surestimons le risque de cancer chez les travailleurs, car on utilise les taux d'induction moyens tout en sachant que ces taux sont plus forts chez les enfants et les adolescents.

\section{PORTÉE DE CES ESTIMATIONS DES RISQUES}

Depuis un demi-siècle, les scientifiques n'ont cessé d'affiner et de quantifier petit à petit le maximum d'informations sur la nature et l'ampleur des dangers qui peuvent résulter d'une exposition à de faibles doses. Que pouvons-nous attendre des enquêtes épidémiologiques et de la recherche fondamentale dans les 10 années à venir? Je ne pense pas que l'on puisse en espérer une découverte majeure. En ce qui concerne les rayonnements à TEL élevé, à savoir les particules lourdes, les évaluations actuelles semblent assez précises. En revanche, dans le cas des rayonnements à TEL faible soit les rayons $\mathrm{X}$ - elles surestiment probablement le risque de deux à cinq fois. Nous pouvons souhaiter que des preuves viennent étayer la théorie de la relation polynomiale du second degré et permettent de chiffrer les coefficients. Mais, même un résultat aussi remarquable ne modifierait guère le problème de la radioprotection. Supposons, par exemple, que l'on parvienne à évaluer que le risque réel par rem est en réalité de $0,70 \cdot 10^{-4}$ au lieu du chiffre de $1,3 \cdot 10^{-4}$ proposé aujourd'hui. Je ne pense pas que les principales recommandations s'en trouveraient changées ni que le public accepterait mieux le risque des radiations pour autant.

En fait, deux conclusions se dégagent de ce survol des problèmes soulevés par l'évaluation des dangers de la radioactivité : la première est que les risques étudiés sont aléatoires, à savoir probabilistes, par nature. Nous avons affaire à une situation où il est possible qu'il n'existe pas de seuil, ce qui signifierait que toute dose d'irradiation, aussi faible soit-elle, comporte un certain risque. Dans ces conditions, la politique qui consiste à abaisser la radioactivité à un niveau " aussi faible que possible » est justifiée. Toutefois, il faut souligner que ce n'est pas une situation exceptionnelle. La plupart des risques professionnels ou des dangers naturels sont aussi aléatoires, de même que les accidents de la circulation. De plus, sans chercher à minimiser les conséquences de l'énergie nucléaire, il faut rappeler qu'elle n'est pas la source principale de radioactivité à

voL. $15-\mathrm{N}^{\circ} 1$ 
laquelle l'homme soit exposé. L'irradiation médicale entraîne des doses bea 1 coup plus fortes [16]. Dans les deux cas, nous devons poursuivre nos efforts en vue de réduire au minimum l'irradiation lorsqu'elle s'impose, et de l'éviter chaque fois qu'elle est inutile [17].

La deuxième conclusion est que le travail considérable que tous les radiobiologistes du monde ont fourni au cours des 10 dernières années a été payant. Les radiologistes ont été, sans nul doute, les premiers à évaluer quantitativement les risques professionnels. Signalons, au passage, que leur action a eu pour conséquence malheureuse d'attirer l'attention du public sur ces risques. Cependant, il serait temps que d'autres scientifiques et groupes industriels suivent cet exemple. Les produits chimiques présentent des risques de cancer et de mutation génétique bien plus graves et plus difficiles à calculer que ceux de la radioactivité. L'expérience collective des radiobiologistes qui ont calculé les risques et mis au point des normes indiquent la voie qui devrait être suivie. Or, des recherches dans ce domaine devraient, bien plus qu'en radioprotection, contribuer à améliorer l'état sanitaire de la population.

En radioprotection, on a surtout insisté jusqu'à présent sur les tumeurs malignes et autres effets létaux. La C.I.P.R., sous l'influence de Sir Edward Pochin, a récemment fait un pas en vue de mettre au point un indice du dommage qui couvrirait non seulement les décès mais aussi toutes les maladies et tous les accidents, mortels ou non, consécutifs à une irradiation professionnelle, exprimerait la réduction de la longévité ainsi que la durée de l'incapacité professionnelle et, enfin, tiendrait compte des diverses tensions, angoisses et privations liées à ces conditions [8]. Cette tâche est, sans nul doute, considérable, mais devrait, elle aussi, être payante. Une fois de plus, les radiobiologistes sont probablement les seuls qui soient capables de concevoir un modèle qui serait ensuite applicable à d'autres risques professionnels.

\section{NORMES DE RADIOPROTECTION}

L'évaluation du risque, ou du détriment, n'est que la première étape de l'établissement des normes de radioprotection. La seconde, aussi importante que la première, consiste à déterminer le dommage acceptable.

Alors que, dans la première étape, on part de faits, ou de données scientifiques, dans la seconde, toute décision implique un jugement de valeur et est donc contestable. Pour ma part, j'estime qu'il serait plus approprié de laisser ce choix aux représentants de la communauté toute entière plutôt qu'aux seuls radiobiologistes. Quoi qu'il en soit, l'expérience acquise dans d'autres domaines de la médecine du travail et des affections dues à l'environnement laisse à penser que si les scientifiques n'avaient pas accepté cette responsabilité, personne ne l'aurait assumée, ou encore que les décisions auraient eu un caractère subjectif et émotionnel.

Il existe deux méthodes pour établir le niveau de dommage tolérable. La première consiste à mettre en balance les inconvénients d'un côté et les bienfaits de l'autre, la seconde à comparer les risques présentés par les rayon- 
nements aux dangers spécifiques d'autres industries considérées comme sûres ou à ceux présents dans la vie quotidienne.

La comparaison des risques et des avantages a été très en vogue pendant quelques années. L'idée était qu'à tout risque lié à une exposition devait correspondre un bénéfice qui justifierait qu'on accepte ce risque, et qu'il fallait rechercher un équilibre coût-bénéfice optimal. Ce type d'analyse peut aider les techniciens à prendre des décisions. Mais cette méthode est semée d'embûches. Les risques et les bienfaits ne sont pas de même nature : on a, d'un côté, une maladie causée à l'homme et, de l'autre, une dépense d'argent. Il est difficile de les évaluer en utilisant la même unité. Le sacrifice d'une vie humaine est une notion que le grand public n'accepte pas facilement, car une vie n'a pas de prix. On peut, dès lors, difficilement éviter qu'interviennent dans une comparaison comme celle-ci des considérations subjectives ou des facteurs émotionnels. Aucun raisonnement fondé sur une comparaison des risques et des avantages ne peut obtenir le consentement du public. Il peut même être nuisible dans la mesure où il éveille certaines craintes. Cette méthode qui met l'accent sur les dangers potentiels peut finalement susciter une politique de refus de tout risque.

La comparaison entre le risque d'irradiation dans le cadre d'une activité professionnelle et les risques dans d'autres industries ou activités humaines semble être une tactique plus efficace et plus pratique $[12,13]$. En ce qui concerne les rayonnements, les risques sont évalués en fonction d'hypothèses pessimistes. Dans d'autres professions, les accidents ou les maladies sont effectivement constatés, mais les effets différés, susceptibles d'apparaître longtemps après la retraite, peuvent être en grande partie négligés ou ignorés. Malgré ce handicap, les tableaux montrent que les résultats sont meilleurs dans les métiers impliquant une exposition aux rayonnements que dans les activités professionnelles considérées comme "sans danger » (tableaux I et II). De surcroît, la dose annuelle moyenne est généralement bien en-deçà de la dose maximale admissible de $5 \mathrm{rem}$ par an. Dans les centrales nucléaires et les hôpitaux, elle se situe généralement entre 10 et $20 \%$ de ce niveau (tableau III).

Lorsqu'il s'agit de l'ensemble de la population, l'irradiation naturelle constitue un excellent point de comparaison. Dans la plupart des pays, elle varie d'un facteur 2 au moins d'une région à l'autre sans se traduite pour autant par une différence dans la fréquence des cancers ou des malformations génétiques. Tant que l'irradiation artificielle restera de l'ordre de grandeur actuel, son danger sera vraisemblablement très faible par rapport à celui d'autres activités de la vie courante. Toutefois, il ne faudrait pas négliger la possibilité d'une "potentialisation » d'un facteur cancérogène par un autre. Il sera donc justifié, dans l'avenir, d'étudier l'effet global de tous les facteurs cancérogènes et mutagènes présents dans notre environnement.

J'ai tenté dans cet exposé d'utiliser des arguments objectifs et de m'en tenir aux faits. Pourtant, la perception et l'acceptation du risque devraient être également étudiées lorsque l'on procède à des comparaisons de ce type. Mais c'est là un autre problème que je ne traiterai pas ici car il sera examiné par ailleurs. 
TABLEAU I

\begin{tabular}{|c|c|c|}
\hline & & $\begin{array}{c}\text { Pour } \\
10^{6} \text { pers./an }\end{array}$ \\
\hline Nombre & total de morts (France) .... & 11500 \\
\hline & $\ldots \ldots \ldots \ldots \ldots \ldots \ldots \ldots \ldots \ldots \ldots \ldots$ & 2300 \\
\hline & Tabac $(b) \ldots \ldots \ldots \ldots \ldots$ & 1300 \\
\hline Causes & Alcool $(b) \ldots \ldots \ldots \ldots \ldots \ldots \ldots \ldots$ & 750 \\
\hline de la & Accidents de la route $\ldots \ldots \ldots \ldots \ldots \ldots \ldots \ldots \ldots$ & 285 \\
\hline mort & Maladies professionnelles et accidents du travail & 160 \\
\hline & Accidents dans le cadre des loisirs $\left({ }^{c}\right) \ldots \ldots \ldots \ldots \ldots \ldots$ & 180 \\
\hline Travaille & rs (dose moyenne dans les centrales nucléaires) $\left({ }^{d}\right) \ldots \ldots$ & 50 \\
\hline $\begin{array}{l}\text { Populati } \\
\text { nucléa }\end{array}$ & $\begin{array}{l}\text { dans son ensemble (dose du fait de l'industrie } \\
\text { e) }\left({ }^{d}\right) \ldots \ldots \ldots \ldots \ldots \ldots \ldots \ldots \ldots \ldots \ldots \ldots \ldots \ldots \ldots \ldots \ldots\end{array}$ & 0,1 \\
\hline
\end{tabular}

(a) Incluant les cancers dus au tabac ou à l'alcool.

(b) Incluant les morts dus au cancer et aux autres maladies.

(c) Excluant les morts dus aux accidents de la route et incluant la nage, l'alpinisme, le bateau, l'équitation, le ski, les insolations, etc.

(d) Morts dus aux affections malignes calculées selon l'hypothèse pessimiste d'une extrapolation linéaire.

\section{TABLEAU II}

Un risque de mort de 1 sur 1 million correspond à :

$-650 \mathrm{~km}$ en avion;

$-100 \mathrm{~km}$ en voiture;

- 3/4 d'une cigarette;

$-1,5$ min d'alpinisme;

$-1,5$ semaine de travail dans une usine typique (Royaume-Uni);

$-20 \mathrm{~min}$ de vie à 60 ans;

- Absorption de pillules contraceptives pendant 2,5 semaines;

$-1 / 2$ bouteille de vin;

- Exposition à $10 \mathrm{mrem}\left\{\begin{array}{l}\begin{array}{c}\text { Exposition à la dose maximale admissible } \\ \text { (professionnelle) d'une demi-journée }\end{array} \\ \text { ou } \\ \begin{array}{c}\text { Séjour pendant } 3 \text { ans au voisinage d'une centrale } \\ \text { nucléaire }\end{array}\end{array}\right.$ 
TABLEAU III

ÉTATS-UNIS 1979, D'APRÈS [17]

\begin{tabular}{|c|c|}
\hline & $\begin{array}{c}\text { Dose } \\
\text { (mrem/an) }\end{array}$ \\
\hline \multicolumn{2}{|l|}{ Population dans son ensemble: } \\
\hline - radioactivité naturelle ..................... & 100 \\
\hline - utilisation médicales des rayons $\mathrm{X} \ldots \ldots \ldots \ldots \ldots \ldots \ldots \ldots \ldots \ldots \ldots \ldots$ & 100 \\
\hline 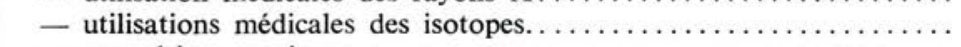 & 20 \\
\hline- retombées atomiques. $\ldots \ldots \ldots \ldots \ldots \ldots \ldots \ldots \ldots \ldots \ldots \ldots \ldots$ & 5 \\
\hline - toute autre forme de radioactivité artificielle $\left({ }^{a}\right) \ldots \ldots \ldots \ldots \ldots$ & 4 \\
\hline \multicolumn{2}{|l|}{ Irradiations dans le cadre de l'activité professionnelle : } \\
\hline - 200000 travailleurs pratiquant des radiographies médicales....... & $300-350$ \\
\hline-200000 personnel pratiquant des radiographies des dents....... & $50-125$ \\
\hline - 100000 personnel médical manipulant des radio-isotopes. . . . . . . & $260-540$ \\
\hline - 30000 personnel travaillant dans l'industrie nucléaire civile...... & $600-800$ \\
\hline - 35000 personnel travaillant sur les navires à propulsion nucléaire & $130-330$ \\
\hline 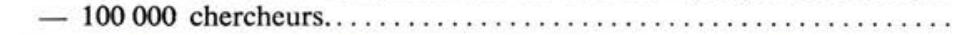 & $130-330$ \\
\hline
\end{tabular}

$\left.{ }^{(}\right)$Incluant l'irradiation due à l'extraction et au traitement de l'uranium, aux centrales nucléaires, aux déchets, etc. L'irradiation due aux centrales nucléaires était dans les pays occidentaux inférieure à 1 mrem en 1977 et sera d'environ 2 mrem en 2000 si $60 \%$ de l'électricité est produite par les centrales nucléaires [16].

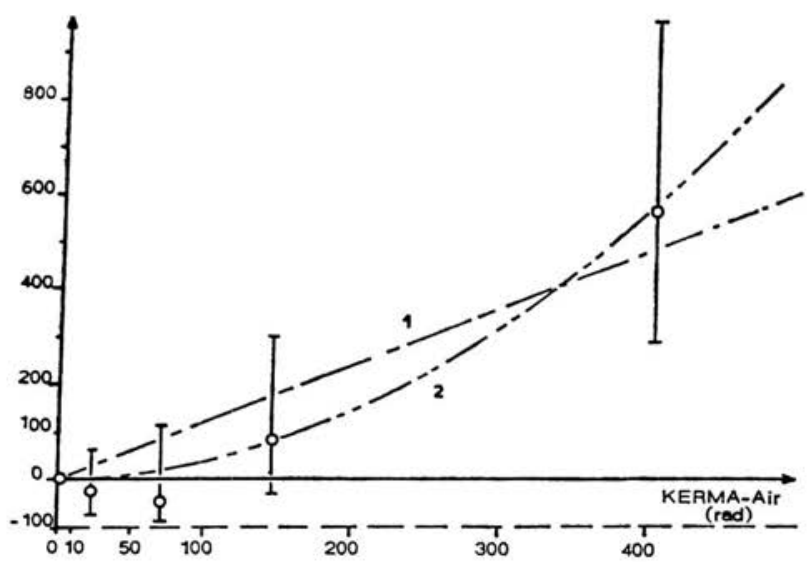

Fig. 1. - Leucémies à Nagasaki. Risque ajouté par l'irradiation. Le risque ajouté est donné pour les individus de chaque échantillon avec un intervalle de confiance bilatéral à $95 \%$. Comme on le voit, les points s'accordent beaucoup mieux avec une relation de type quadratique qu'avec une relation linéaire.

Risque individuel ajouté $r=p-p_{0}\left(10^{-6} / \mathrm{an}\right)$

voL. $15-\mathrm{N}^{\circ} 1$ 


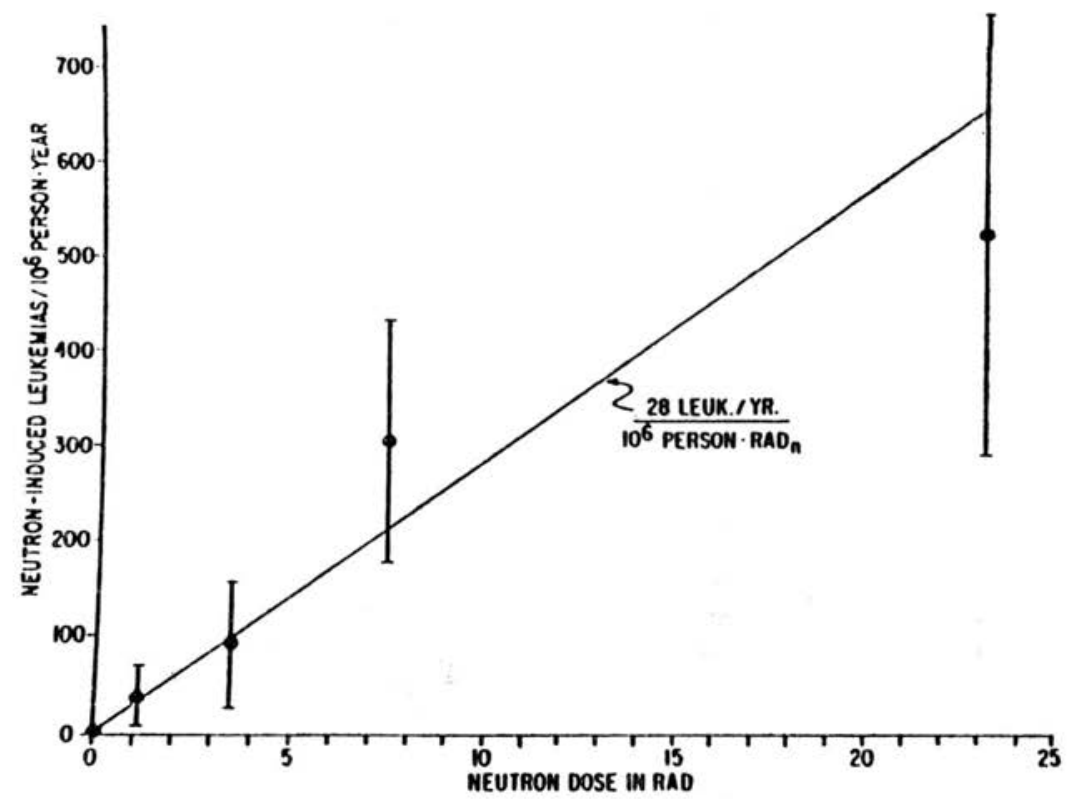

Fig. 2. - Relation dose-effet chez les survivants d'Hiroshima en fonction de la dose neutron qu'ils ont reçue (Rossı, 1978). Comme on le voit, les points suggèrent l'existence d'une relation dose-effet linéaire.

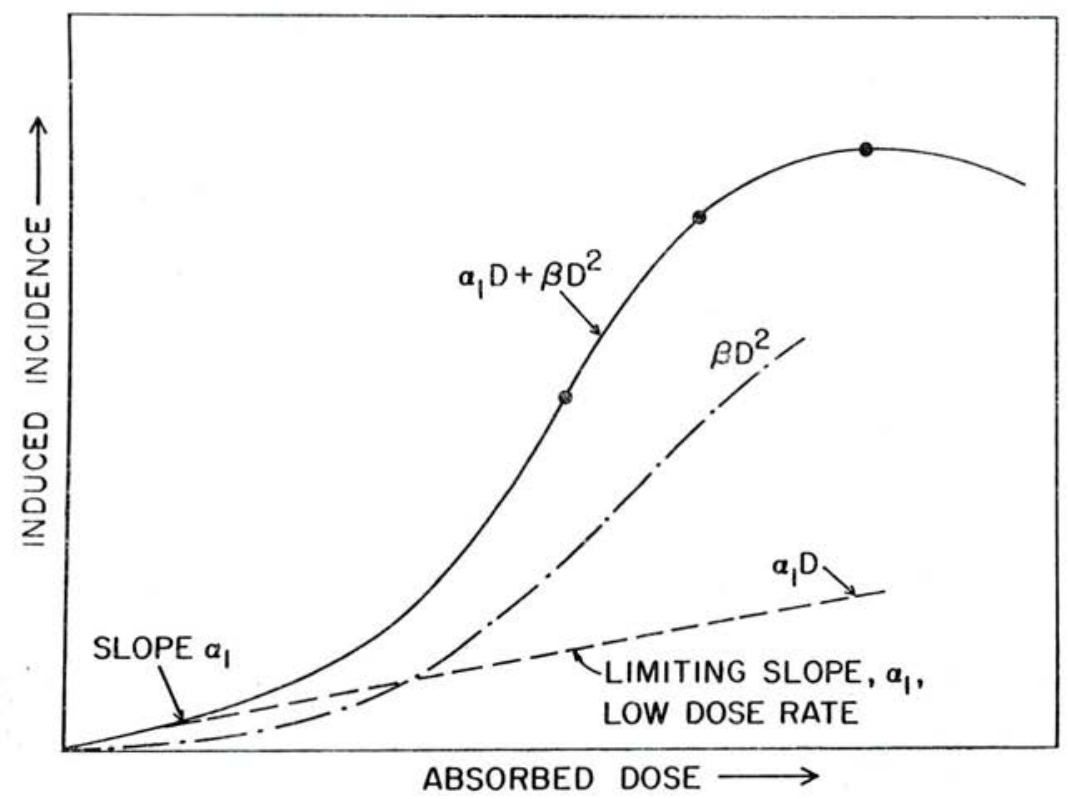

Fig. 3. - Relation dose-effet pour les mutations de la Tradescantia Les points expérimentaux sont en bon accord avec l'hypothèse d'une courbe linéaire quadratique (NAUMAN, 1975). 


\title{
ERRATUM
}

\author{
Radioprotection, vol. 15, 1, 1980
}

Article de M. Tubiana

Les bases biologiques de la radioprotection

Il faut ajouter dans le texte à toutes les références bibliographiques, à partir du numéro 6, une unité, afin de se conformer à la liste bibliographique en fin d'article. 



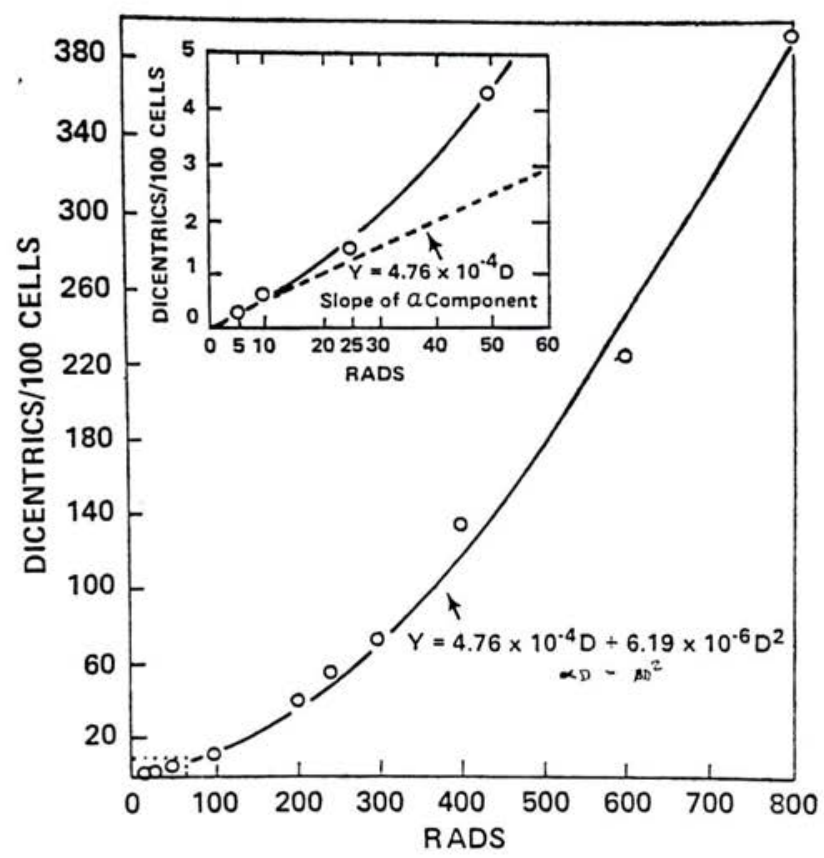

Fig. 4. - Relation dose-effet pour les aberrations chromosomiques radio-induites dans les lymphocytes humains cultivés in vitro. Les points expérimentaux correspondent bien à une relation de type linéaire quadratique (LLYOD, 1975).

\section{BIBLIOGRAPHIE}

[1] Bond V. P., Meinhold C. Rossi H. H. Low-dose RBE and Q for X-ray compared to $\alpha$-ray radiations. Health Physics, 1978, 34, 433-438.

[2] Brown J. M. Linearity vs. non-linearity of dose response for radiation carcinogenesis. Health Physics, 1976, 31, 231-245.

[3] Brown J. M. The shape of the dose-response curve for radiation carcinogenesis. Rad. Res., 1977, 71, 34-50.

[4] Crow J. F. Radiations, environmental mutagens and human life. Can we assess the genetic risk? In: Radiation research, proceedings of the 6 . intern. congress of radiation research, Tokyo, May 1979 (OKadA S. et al., Eds), Tokyo, J.A.R.R., 1979, 70-78.

[5] FINCH C. S. The study of atomic bomb survivors in Japan. Amer. J. Medicine, 1979, 66, 899-901.

[6] Hiroshima and Nagasaki, a review of 30 years study. In: Radiation research, proceedings of the 6. intern. congress of radiation research, Tokyo, May 1979 (OKADA S. et al., Eds), Tokyo, J.A.R.R., 1979, 50-58.

[7] International Congress of Radiology. International recommendations for $X$-rays and radium protection. Br. J. Radiol., 1928, 1, 358. 
[8] I.C.R.P. Publication 26, Recommendations of the International Commission on Radiological Protection, Annals of the I.C.R.P., 1977, 1, $\mathrm{n}^{\circ}$ 3. Pergamon Press, Oxford.

[9] I.C.R.P. Publication 27, Problems involved in developing and index of harm, Annals of the I.C.R.P., 1977, 1, $\mathrm{n}^{\circ}$ 5, Pergamon Press, Oxford.

[10] Leenhouts H. P., Chadwick K. H. An analysis of radiation-induced malignancy. Int. J. Rad. Biol., 1978, 33, 357-370.

[11] Lloyd D. C., Purrot R. J., Dolphin G. W., Dawn Bolton, Edwards A. A., Corp M. J. The relationship between chromosome aberrations and low-LET radiation dose to human lymphocytes. Int. J. Radiat. Biol., 1975, 28, 75-90.

[12] Mancuso T. F., Steward A., Kneale G. Radiation exposures of Hanford workers dying from cancer and other causes. Health Physics, 1977, 33, 369-385.

[13] Pochin E. E. Occupational and other fatality rates. Community Health, 1974, 6, 2.

[14] Pochin E. E. Estimates of industrial and other risks. J. Royal College of Physicians of London, 1978, 12, 210.

[15] SANDERS B. S. Low-level radiation and cancer deaths. Health Physics, 1978, 34, 521-538.

[16] Underbrink A., Kellerer A., Mills R., Sparrow A. Comparison of X-ray and gamma ray dose-response curves for pink somatic mutations in Tradescantia clones 02. Rad. Environ. Biophys., 1976, 13, 295-303.

[17] U.N.S.C.E.A.R. United Nations Scientific Committee on the Effects of Atomic Radiation. 1977 Report to the General Assembly, Sources and Effects of Ionizing Radiation, (U.N. E.77.IX.1), United Nations, New York, 1977.

[18] The effects on population of exposure to low levels of ionizing radiation, report of the Advisory Committee on the biological effects of ionizing radiations (BEIR III), Washington, National Academy of Sciences, 1979. 